International Journal of Wireless \& Mobile Networks (IJWMN) Vol. 5, No. 3, June 2013

\title{
Achieving Transmission Fairness in Distributed Medium Access Wireless Mesh Networks: Design Challenges, Guidelines and Future Directions
}

\author{
Salitha Priyanka Undugodage and Nurul I Sarkar \\ School of Computing and Mathematical Sciences \\ Auckland University of Technology, Auckland, New Zealand \\ Nurul.sarkar@aut.ac.nz
}

\begin{abstract}
Wireless mesh networking gained an international interest over the years as a result to high recognition in the wireless industry as a cost effective, scalable, wider coverage and capacity capable wireless technology. The contention based distributed medium access in wireless networks has advanced not only in supporting the quality of multimedia but also achieving high throughput and to minimize packet delay overheads in legacy systems. Unfortunately, the impact of such enhancement has not been fully justified with mesh network environments yet. The medium access frames are required to be contended over multi-hops to overcome the challenges of improving overall system performance through concurrent transmissions. The goal of this paper is to discuss the issues and challenges of transmission fairness and the effect of concurrent transmission on system performance. To mitigate transmission fairness issues, we review existing open literature on mesh networking and provide guidelines for better system design and deployment. Finally, we conclude the paper with future research directions. This study may help network designer and planner to overcome the remaining challenging issues in the design and deployment of WMNs worldwide.
\end{abstract}

Keywords

Wireless Mesh Networks (WMN), Frame Aggregation, Block Acknowledgement (BA), Reverse Direction Grant (RDG), Carrier Sensed Threshold (CST)

\section{INTRODUCTION}

The Wireless Mesh Network (WMN) is a set of wireless nodes where each node can communicate directly with one or more peer nodes. WMN has been standardized by IEEE 802.11 Task Group "s" to develop a set of standards for WMNs under the IEEE 802.11s. Further the IETF (Internet Engineering Task Force) had also setup wireless mesh networking called Mobile Ad-Hoc Network (MANET) with a separate set of standards. Both MANETs and WMN nodes exploit the redundancy of connected nodes and have the ability of self-organize, self-discover, self-heal, and self-configure. However, in real-world applications, MANETs are implemented with mobile and more power constrained nodes, and the infrastructure is less self-organized. In contrast, WMNs are typically a collection of more organized stationary nodes and may use multiple radios for the purpose of wireless mesh backhauling for Wireless Local Area Network (WLAN) with one radio and the other radio for Access Point (AP) functionality [1]. Although WMNs could extend the wireless coverage as a cost-effective backhaul solutions it has many 
International Journal of Wireless \& Mobile Networks (IJWMN) Vol. 5, No. 3, June 2013

challenges, especially when increasing the per user data rate of multiple concurrent sessions between multi-hop mesh nodes in serving as backhaul WLAN technologies. These challenges are as a result of 802.11's shared medium access constrains in achieving transmission fairness, especially in multi-hop networks.

In this paper we address some of the key issues of such constrains and provide guidelines for network researchers and designer for efficient system design and deployment of such system. The remainder of this paper is organized as follows. In Section 2 we highlight 802.11 Physical layer (PHY) and Medium Access Control (MAC) layer standards in WMNs focusing on distributed medium access protocols. The issues and challenges in designing WMNs are also discussed. Section 3 presents WMN architecture highlighting the transmission fairness issues in a multi-hop contention based shared medium access. In Section 4, we discuss MAC enhancements for multi-hop WMNs medium access efficiency. Section 5 discusses transmission fairness focusing on optimum concurrent transmission in a mesh network. An amendment to the shared MAC with a reverse direction MAC frame pull mechanism to optimize concurrent transmission is also discussed. In Section 6, we present guidelines for WMN design and deployment and future research directions. Finally, a brief discussion in Section 7 concludes the paper.

\section{WMN Design Challenges in Distributed Medium ACCeSS}

One of the primary objectives of 802.11s WMN standardization was to define the 802.11 PHY and MAC layers to create a Wireless Distribution System (DS) which is capable of automating topology learning and wireless path configuration for self-learning, self-forming and self-healing wireless paths. The standard defines dynamic and radio-aware path selection mechanism to delivery of data on both single-hop and multi-hop networks. Any wireless node complying with these functionalities are said to be wireless mesh capable nodes which forms a WMN or a mesh cloud. One of the key issues in WMN standardization is the adaptation of legacy distributed medium access schemes to share the medium which has inherent unfairness in achieving concurrent transmissions between mesh nodes in a multi-hop mesh network. However, it is important that WMN standards should address these challenging issues without compromising the compatibilities of WMNs to continue to evolve as a cost-effective backhauling technology for WLANs [2] [3] [4].

\subsection{Mesh Network PHY and MAC layer Standards}

The IEEE 802.11 PHY and MAC Layer standards were first introduced in 1997. Since then multiple standards had evolved under different IEEE Task Groups as "a" (TGa) and "b" (TGb) in 1999, "g" (TGg) in 2003 and "n" (TGn) 2007. These WLAN standards had evolved with the number of enhancements into the PHY and MAC layers mainly to improve raw data speed and propagation range while maintaining backward compatibility with the previous standards. Consequently, the 802.11g APs are backward compatible in connecting 802.11b Stations (STAs). Similarly IEEE $802.11 \mathrm{n}$ APs are backward compatible in communicating to $802.11 \mathrm{a} / \mathrm{b} / \mathrm{g}$ STAs. A WLAN operating in multi-mode supporting more than one mode is said to be in "mixed mode" whereas a WLAN is said to be operating in "Green field" if all STAs only support native highest performing mode. The most capabilities of Green field operation are compromised when operating in mixed mode. In theory 802.11 s could operate on any 802.11 PHY layer standard 
International Journal of Wireless \& Mobile Networks (IJWMN) Vol. 5, No. 3, June 2013

supporting either mixed mode or green field but it is sensible for all mesh nodes to be deployed in a same mode (e.g. green field network) for greater performance [5].

Figure 1 shows 802.11 MAC protocol structure. The MAC layer defines the data link between two mesh nodes and exchanges MAC Service Data Units (MSDUs) packed into MAC Protocol Data Units (MPDU) and carried over the PHY Protocol Data Unit (PPDU) as per the original 802.11 MAC standards. The main concern observed in a wireless mesh is that the standard requires every successfully non multicast and broadcast frames received at each mesh node to be acknowledged causing considerable packet delays in multi-hop communications (a frame needs to cross multiple hops in reaching the destination) [6] [7].

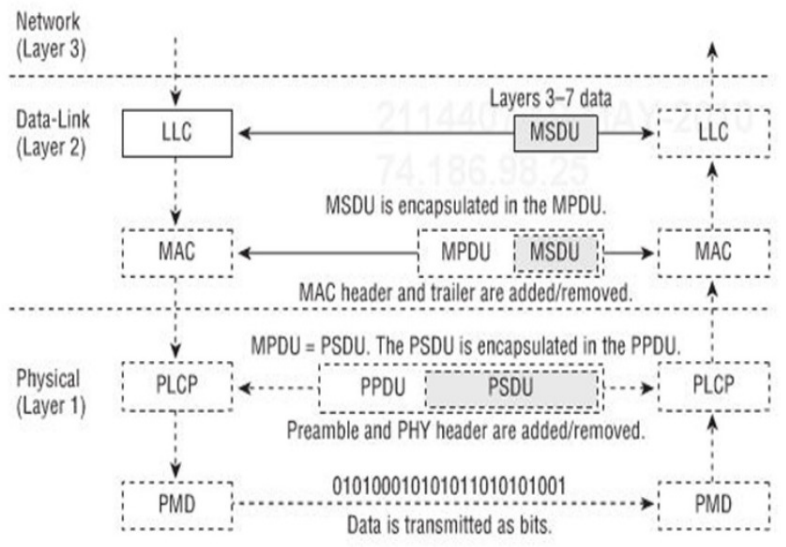

Figure1: Media access control (MAC) protocol structure.

\subsection{WMN MAC Layer Protocol Design Challenges}

IEEE 802.11 medium access protocol is based on Carrier Sense Multiple Access with Collision Avoidance (CSMA/CA) to avoid frame collisions in a shared wireless channel. This medium access layer is similar to IEEE 802.3 wire-line medium access which is based on Carrier Sense Multiple Access with Collision Detection (CSMA/CD) where medium access coordination mechanism is to detect rather than avoid collisions only. CSMA/CD is not suitable for wireless network because network interface cards cannot transmit and listen on the same wireless channel simultaneously. The Receiver ( $\mathrm{Rx}$ ) must receive the incoming frame fully before the wireless interface could switch from "receive" mode to "transmit" mode to transmit a frames which makes CSMA/CA ideal in wireless contention based shared access. The idea of carrier sense is listen before transmit in assessing nearby node engage in transmission. The Transmitter (Tx) will refrain from transmission if received energy level at a Tx at any time slots duration higher than a fixed Carrier Sensed Threshold (CST) to avoid collision [8] [9]. Although this is acceptable in an AP centric WLAN implementation, it is a major concern in WMNs where exposed mesh nodes suspend any concurrent transmission to avoid collisions. To overcome this problem it is possible to separate mesh nodes so that they do not necessarily exposed to each other. However this may lead to increased collisions at the Rx end if a Transmit mesh node estimates a lower energy level from another hidden mesh node that could transmit at the same time slot. This is known as Hidden Station Problem which is a well-known issue in CSMA/CA medium access. The IEEE has standardized the 802.11 Distributed Coordination Function (DCF) for contention based medium access minimizing the hidden station problem by either 2-Way handshaking where each 
MAC frame is acknowledged by an acknowledge frame (ACK) or 4-Way handshaking with an additional contention free medium protection called RTS/CTS handshaking or both by providing any hidden nodes access the shared channel. In fact, as shown in Figure 2 RTS/CTS is a virtual carrier sense mechanism to block any attempt to transmit by any exposed nodes for a specified duration called Network Allocation Vector (NAV) exclusively allocating the channel to the nodes that raise the RTS and CTS as shown in Figure 2. However, a complete elimination of hidden mesh nodes as well as exposing all mesh nodes in a WMN refraining concurrent transmission between mesh nodes could be challenging, as it leads to unfairness in sharing channel for multi-hop transmissions [10]. The multi-hop network throughput scenario/analysis is discussed next.

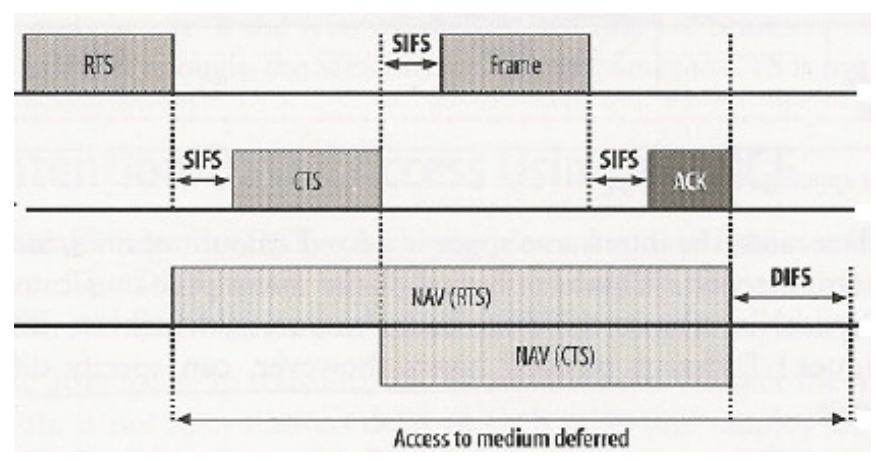

Figure 2: RTS/CTS and NAV timing diagram.

\subsection{Multi-Hop Network Throughput Analysis}

To eliminate the hidden station problem and to avoid collisions, mesh nodes must maintain received power levels within carrier sensed threshold (CST). However this could prevent the nodes that are exposed utilizing the medium for concurrent transmission due to the DCF contention access scheme which is called Exposed Station Problem which is a major barrier to exploit concurrent transmissions in multi-hop mesh networks. Inability to perform concurrent transmission between mesh nodes in a multi-hop network would increase the MAC frame transmission waiting times with the increase of mesh node density resulting in rapid throughput degradation. In other words, the balance between hidden and exposed nodes is crucial in optimizing the concurrent communications in a WMN [11] [12] [13].

When all mesh nodes are in the same collision domain and if $\mathrm{N}$ numbers of nodes are exposed to each other, the probability of successful frame transmission would be $1 / \mathrm{N}$. Assuming $\mathrm{N}$ number of hops or $\mathrm{N}+1$ number of nodes in an exposed collision domain and no packets are losses at relay nodes between source to destination as well as negligible propagation time between nodes then the single-hop normalized end to end throughput would be:

$$
\frac{1}{N+(N-1) \frac{T_{t}}{T_{p}}}
$$

Where $T p \quad=$ Time to Transmit / Receive payload at a node

$T i=$ Intermediate relay node transmission latency 
International Journal of Wireless \& Mobile Networks (IJWMN) Vol. 5, No. 3, June 2013

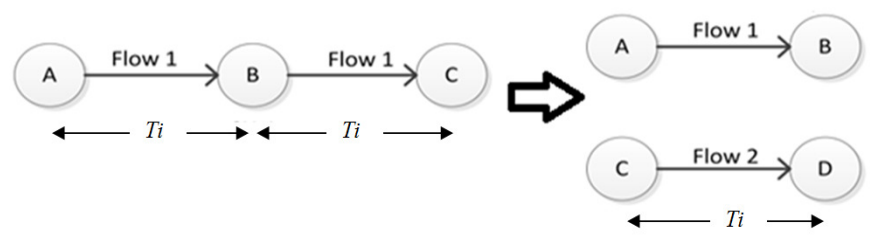

Figure 3: Multi hop concurrent transmission delay

The above expression shows that the packet delay overheads could be minimized to improve the raw data transmission efficiency by minimizing $T i$ which will in return increase the throughput between mesh nodes. Applying this model to the concurrent transmission scenario as shown in Figure 3 for a contention based shared medium access scheme where $T p$ is unbounded, indicating that constrains in concurrent transmission between multi-hops could limit the per user throughput when multiple user consume network bandwidth [14] [15] [16] [17].

\subsection{IEEE 802.11 DCF Access Mechanism}

In 2-way handshaking (Figure 4) when the medium is idle and the nodes contending for the medium will access the channel immediately after the period of Short Inter-Frame Space (SIFS). If expecting any acknowledgement frames for prior transmissions and wait further duration up to DCF Inter-Frame Space (DIFS).

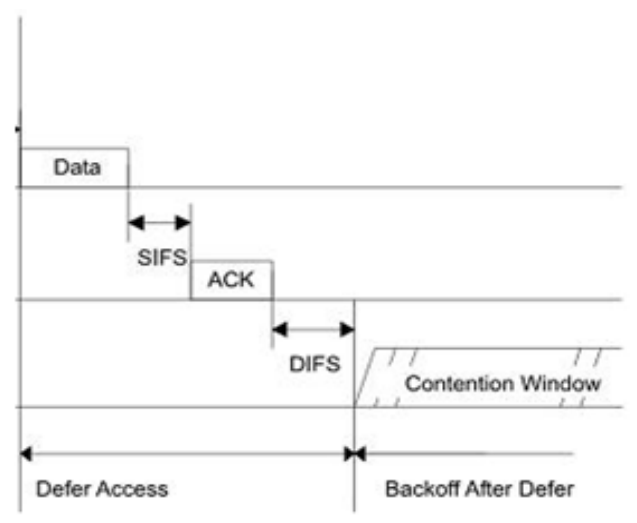

Figure 4: Inter-frame spacing and back-off.

If the medium is not idle, nodes will continue to wait a random back-off period set up in the Back-off Counter (BC). The node transmits when BC expires to minimize any possible collisions in transmissions. $\mathrm{BC}$ is a uniformly distributed random number between 0 and a Contention Window $(\mathrm{CW})$ defined. The $\mathrm{CW}$ size is initially assigned $\mathrm{CW}_{\min }$, and increases by doubling $\mathrm{CW}$ with an upper bound of $\mathrm{CW}_{\max }$ when collision is experienced but every successful transmission will reset $\mathrm{CW}$ back to $\mathrm{CW}_{\text {min. }} \mathrm{CW}$ size is measured in terms of slot time which is defined for different 802.11 PHY standards [12] [2]. Figure 4 illustrates the basic concept of inter-frame spacing and back-off mechanisms.

These inter-frame spacing and random back-off introduce delay overheads where medium left unutilized before each transmission but built into the DCF scheme to minimize possible collisions [9]. Figure 5 illustrates the overheads associated with 2-way handshaking. This delay overhead is even worst with 4-Way handshaking where additional RTS/CTS NAV delays further 
International Journal of Wireless \& Mobile Networks (IJWMN) Vol. 5, No. 3, June 2013

contribute to longer underutilised medium assuring the medium protection for transmission without collision at a cost of overall raw data rate degradation. In fact 4-Way handshaking or RTS/CTS is usually recommended to be used only when long frames are to be transmitted where a retransmission degrades system performance in case of a frame losses due to collisions [12].

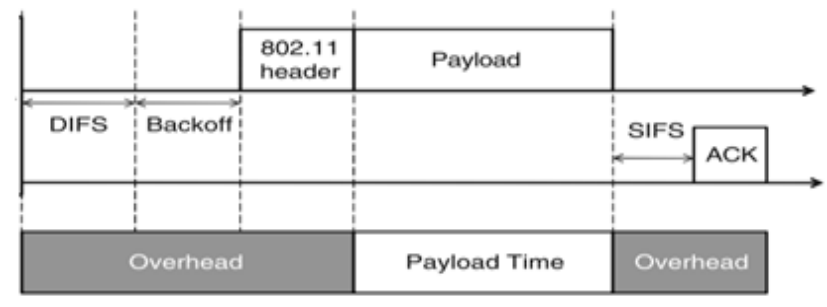

Figure 5: Overheads in 2-way handshaking.

\section{Wireless Mesh Network Elements}

Figure 6 shows the architecture of a typical wireless mesh network. The 802.11 standard defines the WLAN Basic Service Set (BSS) where a set of WLAN STAs that are associated to an AP or each other in an ad hoc manner. Similarly in a WMN, the mesh nodes called as Mesh Point (MP) are associated to each other based on the 802.11s standardized Mesh Basic Service Set (MBSS). In other words the MBSS is a set of MPs that are associated to each other forming a transparent single broadcast domain mesh cloud. However, unlike WLAN BSS STAs the MPs in a MBSS has the relaying capability and MPs could exchange MAC frames over multiple wireless hops by maintaining established mesh links with peering MPs in its neighborhood. The MBSS mesh topology formed by MPs searches for potential MPs present in the neighbourhood by either active scanning or passive listening over air waves and exchanging the Mesh Profile which consisting of a Mesh ID, Path selection protocol identifier, and Link metric identifier. In fact, the Mesh Profile that matches each other got associated them-self forming partial or a full mesh topology. Once associated the MPs establish mesh links and continue to exchange beacons frames for topology maintained and concatenated set of mesh links established via reachable MPs maintained mesh paths in a mesh topology [7] [6].

\subsection{WMN Mesh Functionality and Routing}

MPs in a WMN could have one or multiple optional functions other than the mandatory mesh function, such as the AP function which allows an MP to function as an AP to connect 802.11 WLAN STAs and such a mesh node is called a Mesh Access Point (MAP). A MP that could translate 802.11s MAC frames to 802.11 WLAN MAC frames is called a Mesh Gateway (MG). Having gateway functionality and an MG may have external gateway functionality as well to connect an MP to an external 802.3 LAN or wired backhaul such an MP is called a Mesh Portal Point (MPP) as illustrated in Figure 6. 
International Journal of Wireless \& Mobile Networks (IJWMN) Vol. 5, No. 3, June 2013

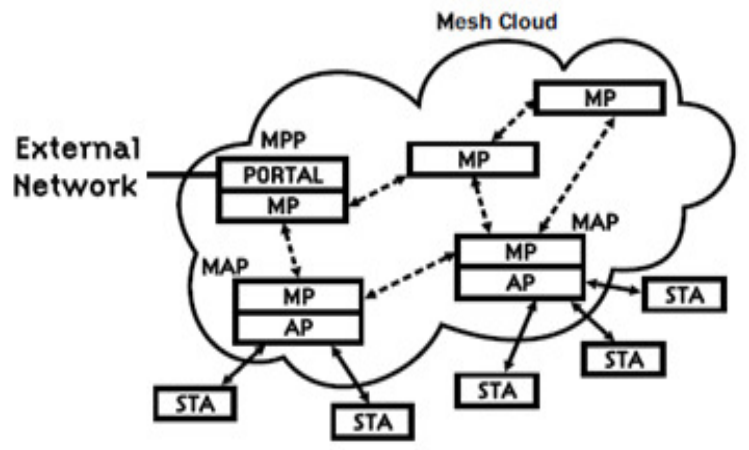

Figure 6: Wireless mesh network topology.

The MPs learn the mesh topology through routing protocols and an interesting feature in $802.11 \mathrm{~s}$ WMN standard is that the definition of its own routing protocols for frame forwarding and path selection in the MAC layer itself without depending on network layer or usual TCP/IP routing Protocols.

The IETF routing and forwarding standard for MANET called Hybrid Wireless Mesh Protocol (HWMP) which provides both on demand routing with Ad-hoc On-demand Distance Vector (AODV) and proactive tree-based routing with Optimized Link State Routing (OLSR) is used in 802.11s WMNs as well. Although WMN framework allows multiple routing protocols to be implemented in a MANET and only one of them could be active in a Mesh cloud [8] [4].

\subsection{Spatial Bias Multi-Hops WMN}

In a multi-hop mesh topology, a user performance depends on the number of hops the frame had to travel in reaching the destination. Higher the number of hop counts, the lower the overall throughput achieved due to contention overheads at each hope resulting unfairness in spatial resources use for an MP which has higher number of hops to the destination. The scenario called spatial bias where more the mesh hops in a mesh path, higher the frames affected which is scalability concern in designing WMN. Research has shown that the bandwidth starvation due to spatial bias in multi-hop could be optimized by dynamically adjusting the packet size and the minimum contention period based on congestion experienced due to spatial bias [6].

\section{MAC ENHANCEMENT SCHEMES}

Figure 7 shows the access control mechanism based on arbitration inter-frame space (AIFS) in EDCA. The 802.11standards had not considered priority base Quality of Service (QoS) and capable of serving only best effort delivery data over WLANs. But with the increasing demand for carrying multimedia traffic over WLANs, the IEEE to form separate Task Group "e" (TGe) introducing standards for Wireless Multimedia (WMM) under the 802.11e standard which extend DCF with QoS capabilities. In the 802.11e standard, different traffic types are classified based on 8 different priority values mapped onto the 4 FIFO queues, called Access Classes (ACs) where each $\mathrm{AC}$ behaves like a virtual node. This WMM medium control coordination scheme is called Enhanced Distributed Channel Access (EDCA) and the contention time DIFS is defined for each corresponding traffic AC as the AIFS as illustrated in Figure 7 [18] [13]. 
International Journal of Wireless \& Mobile Networks (IJWMN) Vol. 5, No. 3, June 2013

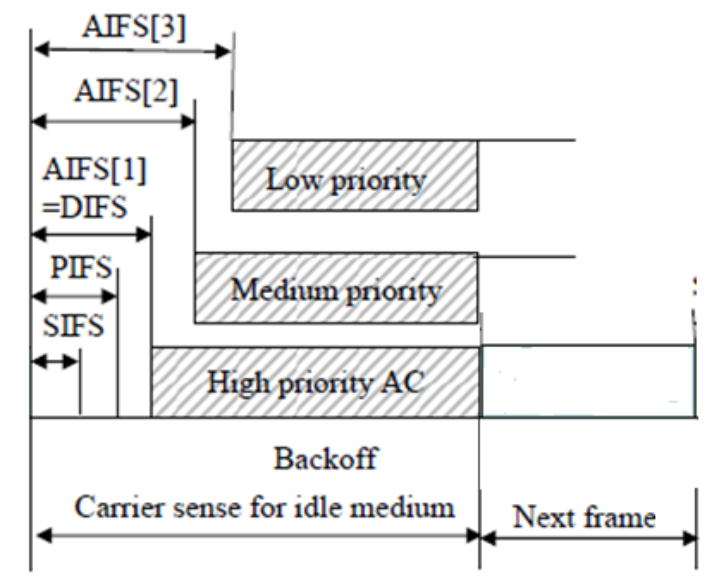

Figure 7: Access control based AIFS in EDCA.

Higher priority traffic category will have a shorter AIFS than a lower priority traffic category which means lower priority traffic must wait longer time than high priority traffic before accessing the medium. Although the probabilistic priority mechanism for allocating bandwidth based on traffic categories has no guarantees of delivery between MPs in a mesh cloud, the EDCA is the mandatory medium access scheme in 802.11s WMNs. As a result 802.11s standard specifies another medium access scheme called Mesh Deterministic Access (MDA) as an option in the Mesh Coordination Function (MCF). MDA is also a distributed and reservation based deterministic medium access scheme and capable of providing prioritized QoS with delivery guarantees. The advantage of MDA compared to EDCA is that the mesh nodes could negotiate a periodic transmission opportunity for collision free transmissions. However, MDA capable mesh nodes need to be synchronized each other and therefore it becomes more complex due to the ad hoc nature of the mesh topology. Further in 802.11s based WMNs, synchronization is optional due to its distributed nature and not all mesh nodes are required to participate in the MDA scheme which could impact the presence of contention from non-MDA mesh nodes in the neighbourhood [19] [17] [20]. The various MAC enhancement mechanisms for WMNs are discussed next.

\subsection{Transmission Opportunity and Frame Aggregation}

IEEE 802.11e WMM standard also adds additional MAC enhancements such as aggregating frames to be transmitted during the opportunity gained by contention scheme which is named Transmission Opportunity (TXOP) and Block Acknowledgement (BA). It enables the receiver to acknowledge the successful reception of multiple frames using a single BA frame. TXOP is a bounded time interval defined by a maximum duration in which a series of frames are transmitted. TXOP Limit, which depends on the AC, is the maximum time a node could hold a channel after a successful contention. Frame aggregation concept allows Aggregated MAC Service Data Unit (A-MSDU) to be sent to the same receiver concatenated into a single MPDU and transmitted either when transmit queue reaches the maximal A-MSDU threshold or any frame timeout condition. The BA contains a bitmap to selectively ACK individual frames in an aggregated frame burst allowing a block of frames separated by an inter frame spacing of SIFS with same AC to be transmitted without waiting for acknowledgment. A-MSDU transmitted is followed by a BA Request (BAR) frame to enquire which frames have been received 
International Journal of Wireless \& Mobile Networks (IJWMN) Vol. 5, No. 3, June 2013

successfully which is answered with a BA frame for every successful frame delivery [5] [21] [22] [23].

\subsection{IEEE 802.11n MAC Enhancements}

IEEE 802.11n WLAN standard added further enhancement for the frame aggregation with another level of aggregation called Aggregated MAC Protocol Data Unit (A-MPDU), aggregating MPDU sub frames to a single PHY frame. Unlike A-MSDU there is no waiting time for an A-MPDU and the number of MPDUs aggregated depends on the number of frames in the transmit queue at the time of gaining the TXOP. MSDUs within an A-MSDU are addressed to the same receiver whereas MPDUs within an A-MPDU need not be to the same receiver. A blend of both A-MSDU and A-MPDU over two stages will maximize throughput efficiency. Further BAR is made optional and Rx could respond with BA after each aggregated frame without waiting for a BAR which removes the BAR overhead and eliminate the possibility of retransmission. This could be any failure to receive BAR and allows multiple aggregated frames to be acknowledged by a single BA. BA could be either expected immediately as a response to the BAR or could be a delayed BA [24] [25] [26].

Another medium access enhancement introduced in $802.11 \mathrm{n}$ is the reduced and zero inter-frame spacing (RIFS and ZIFS) to minimize the overhead between frames. Inter frame spacing is required within TXOP between frames and between the last frame and BAR. This is reduced from SIFS to RIFS where (RIFS $<$ SIFS) between multiple aggregated frames or completely removed which means RIFS $=$ ZIFS eliminating the overhead due to inter-frame spacing resulting more bit transmission using TXOP [5] [27] [28].

\subsection{Reverse Direction (RD) Flow}

IEEE 802.11n standard has also been enhanced frame aggregation called Reverse Direction (RD) flow, which improves the TXOP effectiveness by allowing frame transfer from responder to the originator during originator's TXOP. RD flow initiates with RTS/CTS handshake and the peers make a request inside the RTS/CTS NAV duration. RD flow requires the TXOP originator to grant permission to the responder to send data frames aggregated in the reverse direction while being responsible for channel ownership. Gain in throughput performance would be achieved in RD flow by granting responder node to transfer frames without contention related overheads [29] [30].

\subsection{Green Field High Throughput Mode}

IEEE 802.11n standard is backward compatible with previous generations $802.11 \mathrm{a} / \mathrm{b} / \mathrm{g}$ and operates in three modes, namely Legacy Mode, Mixed Mode and Green Field Mode. In Legacy mode, frames are transmitted in the legacy 802.11a/g MAC format frames with no 802.11n MAC features. In the Mixed Mode, $802.11 \mathrm{a} / \mathrm{g}$ frames are transmitted with a preamble compatible with the legacy $802.11 \mathrm{a} / \mathrm{g}$ such that it can be decoded by legacy $802.11 \mathrm{a} / \mathrm{g}$ devices while transmitting $802.11 \mathrm{n}$ frames with an initial training sequence format which occupies less air time to reduce per-transmission overheads. Therefore, medium protection RTS/CTS handshaking is required to permit communication with legacy stations to ensure legacy devices sense the channel busy state. Thus, $802.11 \mathrm{n}$ devices have to pay significant throughput penalties when legacy devices are served in mixed mode. The Green Field mode is exclusively for 802.11n devices only with high throughput (HT) format preamble is used in MAC frames for HT transmission. If no legacy 
International Journal of Wireless \& Mobile Networks (IJWMN) Vol. 5, No. 3, June 2013

devices served the 802.11n WLANs operate in maximum HT performance then it is said to be a green field network [28] [31] [32] [33].

\section{Transmission Fairness Strategies}

The DCF and the enhanced EDCA contention based distributed access schemes have been successful in all 802.11 standards irrespective of other contention free schemes used in the standards to avoid contention in 802.11n [34].

In contention based distributed medium access schemes for multi-hop networks, nodes are required to accommodate multiple concurrent transmissions. It is important to distribute/separate the nodes to multiple collision domains so that the nodes can be exposed in that collision domain. To carry frames across the collision domains, an overlap between collision domains is required where a node within overlapping area would be the transit node for interconnecting two or more collision domains [35] [36] [37].

Table 1 compares the four main distributed medium access mechanisms for WMNs. The comparison is based on various factors, including IEEE standards, MAC type, operating mode, frame aggregation, support for block Ack (BA), reverse direction (RD), and NAV, and priority.

Table 1: Comparison of distributed medium access schemes

\begin{tabular}{|c|c|c|c|c|}
\hline Scheme & DCF & EDCA & MDA & HT EDCA \\
\hline IEEE Standard & $802.11 \mathrm{a} / \mathrm{b} / \mathrm{g}$ & $802.11 \mathrm{e}$ & $802.11 \mathrm{~s}$ & $802.11 n$ \\
\hline MAC type & Contention & Contention & $\begin{array}{l}\text { No } \\
\text { contention }\end{array}$ & Contention \\
\hline $\begin{array}{l}\text { Mixed mode } \\
\text { supported }\end{array}$ & Yes & Yes & Yes & Yes \\
\hline $\begin{array}{l}\text { Frame } \\
\text { aggregation }\end{array}$ & No & Yes & Yes & Yes \\
\hline Support BA & No & Yes & Yes & Yes \\
\hline $\begin{array}{l}\text { Support for } \\
\text { Reverse } \\
\text { Direction flow }\end{array}$ & No & No & No & Yes \\
\hline RIFS/ZIFS & No & No & No & Yes \\
\hline $\begin{array}{l}\text { Support NAV } \\
\text { Protection }\end{array}$ & Yes & $\begin{array}{l}\text { Only Long } \\
\text { frames }\end{array}$ & Yes & $\begin{array}{l}\text { Mixed } \\
\text { mode }\end{array}$ \\
\hline Synchronization & Optional & Optional & $\begin{array}{l}\text { Manda- } \\
\text { tory }\end{array}$ & Optional \\
\hline AC Priority QoS & No & Yes & Yes & Yes \\
\hline $\begin{array}{l}\text { Exposed STA } \\
\text { avoid scheme }\end{array}$ & No & No & Yes & No \\
\hline $\begin{array}{l}\text { Concurrent Tx } \\
\text { scheme for MPs }\end{array}$ & No & No & No & No \\
\hline
\end{tabular}




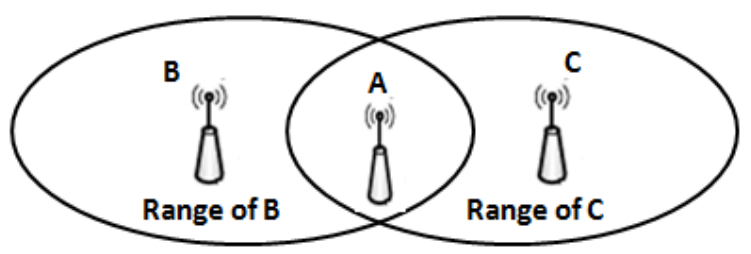

Figure 8: Transit mesh point exposed areas.

A transit node will be exposed to multiple collision domains to contend in both collision domains to have longer span of time for a transmission as shown in Figure 8. This scenario is called "Neighborhood capture problem" where the transit node will hardly find the free medium to access both collision domains [38] [39] [40] [41].

\subsection{Channel Estimated Power Management}

In contention based medium access, the frames are lost as a result of collisions or transmission errors. The transmission errors occurred due to poor channel conditions. The dynamic link adaptation using modulation and coding scheme (MCS) and forward error correction (FEC) compromises the raw data rate by mitigating transmission errors. However, frame losses are normally occurred due to collision at the receiving end when a hidden node attempts to transmit data as illustrated in Figure 9. In a WMN setting, the Tx power and CST levels at each node is a decisive factor in fixing the propagation range of exposed MPs. To select the optimum CST level one could avoid hidden MPs as well as limit the exposed MPs [42] [43] [44].

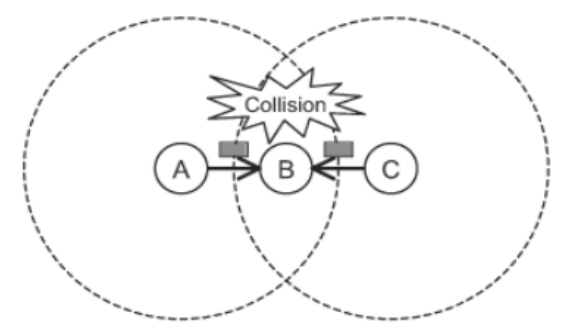

Figure 9: Transit node receiver end collision.

To optimize concurrent transmission between MPs, a strict power management and channel condition estimation at each MP in WMNs is required to ensure MPs are exposed to well manage collision domains.

To analyse MP power management, let us look at the well-known formula for Free Space Power Loss $\mathrm{P}_{\mathrm{L}}$ is given by

$$
\begin{aligned}
& P_{2}(d B)=20 \log _{10}(d)+20 \log _{10}(f)+32.45 \\
& P_{2}(d B)=P_{\text {out }}(d B)+R S S I(d B m)
\end{aligned}
$$

Where $\mathrm{d}$ is the distance (in $\mathrm{km}$ ) between the nodes, $\mathrm{f}$ is the signal frequency in $\mathrm{MHz}$ and $\mathrm{P}_{\text {Out }}$ is the MP Transmit power and the RSSI is the receiving MP Received Signal Strength Indicator in $\mathrm{dBm}$. 
International Journal of Wireless \& Mobile Networks (IJWMN) Vol. 5, No. 3, June 2013

Even at low RSSI levels due to poor channel conditions or high $\mathrm{P}_{\mathrm{L}}$, the MPs could establish a transmission by adopting low bit rate MSC schemes. However, to avoid two MPs exposed to each other it is required to lower the transmit power $\mathrm{P}_{\mathrm{L}}$ to a level such that RSSI level at the receiver is insufficient to establish any transmission even with lowest possible MSC scheme [45] [38].

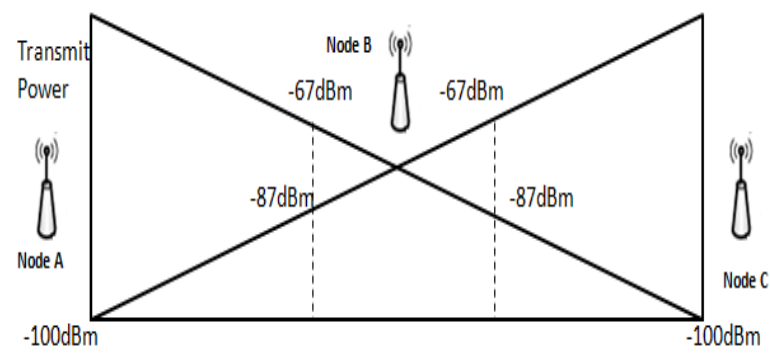

Figure 10: Transmit power management.

Figure 10 demonstrates the transmit power management strategy in WMNs. In 802.11, every frame transmitted is expected to be acknowledged when delivered. If the frame is not acknowledged within a predefine timeout duration, the frame is considered to be lost. The reason for acknowledgement failure which could be either a transmit frame lost due to poor channel conditions or a frame collision that is not known by the sender. However with the introduction of 802.11e MAC enhancements, the BA could be used by the sender to assess the exact reason for an unsuccessful frames delivery. If return indicate many error frames transmitted in the Bitmap, the channel suffers from poor channel conditions. Further if BA is never returned during the BA timeout period then frames could have been collided. This clarity on transmit frames is useful in a WMN to adjust the transmit power levels and CST of an MP to avoid collisions as a result of an optimum collision domain separation [30] [46] [11] [33].

\subsection{Design of RD Pull Collision Avoidance Scheme}

WMN in Greenfield mode will ensure all MPs support 802.11e MAC layer enhancements as well as 802.11n HT features consistently. In a Greenfield mode, a WMN can be implemented in multiple collision domains where each collision domain is interconnected to the neighboring collision domains through one or more transit MPs. This strategy would allow concurrent transmissions without interfering nodes using contention based medium access mechanisms in neighboring collision domains. However, this may lead to transit MP starvation due to neighborhood capture problems. We suggest that the reverse direction (RD) pull mechanism can be used to avoid collisions due to neighborhood capture problems (discuss below). This strategy can be used without modifying the EDCA contention based medium access scheme in WMNs, especially when modeling using a credible simulation package, such as OPNET or ns-2 for performance evaluation [47] [48]. The RD algorithm is described below.

- Neighborhood capture most transit MPs in receiving state and request for RD flow from the sender during sender's TXOP using either CTS frame or BA frame.

- The reverse direction grant (RDG) request could be initiated by the neighborhood captured MPs when its transmit buffer reaches threshold limit. The system may experience collisions when contending for the shared channel or any higher layer strict delivery conditions. 
International Journal of Wireless \& Mobile Networks (IJWMN) Vol. 5, No. 3, June 2013

- Receiver's RDG request is responded by the TXOP's own MP with a RDG allocating any excess TXOP to pull the MAC frames in RD. If the excess TXOP is insufficient to pull all MAC frames waiting to be transmitted, another RDG request can be processed before the end of TXOP.

- If an MP had received a RDG request it will contend to the shared channel to respond with a $\mathrm{RDG}$ in order to pull frame in RD.

Although the RD pull mechanism may halt concurrent transmissions, addressing the neighbourhood captured problems will in fact optimize the concurrent transmission in WMNs [14] [47]. This is an important strategy in achieving high throughput in WMNs. The network design guidelines to improve system performance are discussed next.

\section{DESIGN GUIDELINES AND DisCUSSION}

The three design guidelines for optimizing multi-hop WMNs using concurrent transmissions are discussed below.

(1) Network design using Green field mode: It is important to operate all mesh network MPs in green field mode only. Use all 802.11e wireless multimedia (WMM) and 802.11n HT features to maximize system performance as well as capitalise on novel features such a reverse direction $(\mathrm{RD})$ flow.

(2) Network design by splitting: Split the WMN into multiple collision domains in such a way that MPs in each collision domain can transmit frames within the domain independently to exploit concurrent transmissions. This could be done by varying the transmitter power and CST so that MPs in different collision domains do not associate each other even at the lowest possible modulation and coding scheme (MCS). This strategy of WMN design will optimize network throughput performance.

(3) Network design by exposed collision domain: Ensure that at least two MPs can be exposed to any two collision domains to transit traffic between the two collision domains. This strategy will avoid a single point of failure of a single MP. However, the impact on such a transit MP due to neighbourhood capture problem for being exposed to more than one collision domains needs to be addressed. An effective solution would be to use RD pull mechanism that could pull traffic from transit MPs by granting the RD flow to achieve transmission fairness and optimising concurrent transmissions.

There are many challenging issues in the design, implementation, and deployment of WMNs. Some of the issues are discussed in Section 2 of this paper. Now the question may arise about the better ways of designing WMNs for optimum performance. However, high performance WMN can be (re)design if some obvious guidelines were adopted. In this section we formulated three guidelines for network designers and researchers for optimizing multi-hop WMNs. First, network should be designed using Green field mode only. This means that WMN design using high performance 802.11n devices only. Second, network should be designed by splitting a WMN into multiple collision domains to exploit concurrent transmissions. Third, network should be designed by exposed collision domains. This means that at least two MPs can be exposed to any two collision domains for better system performance. 
International Journal of Wireless \& Mobile Networks (IJWMN) Vol. 5, No. 3, June 2013

\section{CONCLUSION AND Future WorK}

The existing distributed medium access schemes and MAC-layer enhancements for improving concurrent transmission among mesh points (MPs) in WMNs are discussed. The evolution of the 802.11 standards PHY and MAC layers with the assumption that contention based distributed medium access protocols will continue to be the most accepted mechanisms in sharing the medium not only in WLANs but also in multi-hop WMNs. We reviewed existing mechanisms for improving the performance of a typical WMN by concurrent transmission among MPs. These mechanisms can be added to the 802.11 standards especially transmit opportunity (TXOP), frame aggregation, block acknowledgement, reduce inter frame spacing and reverse direction flow. Based on the findings from open literature we identify a high throughput green field WMN separated into multiple collision domains would be the best design strategy to optimize concurrent transmissions in WMNs. We also suggest that reverse direction (RD) pull mechanism can be used to avoid neighbourhood capture issues at a MP specially when handling transit traffic between collision domains. Development of an extensive simulation model of a large WMN with multiple transit mesh nodes handling contention and concurrent transmission is suggested as future work.

\section{Acknowledgment}

This research was supported in part by the Faculty of Design \& Creative Technologies under Summer Studentship grant 2012-2013, Auckland University of Technology.

\section{REFERENCES}

[1] G. R. Hiertz, Z. Yunpeng, S. Max, T. Junge, E. Weiss, and B. Wolz, "IEEE 802.11s: WLAN mesh standardization and high performance extensions," IEEE Network, vol. 22, no. 3, pp. 12-19, 2008.

[2] G. Hiertz, D. Denteneer, L. Stibor, Y. Zang, X. P. Costa, and B. Walke, "The IEEE 802.11 Universe," IEEE Communications Magazine, vol. 48, no. 1, pp. 62-70, 2010.

[3] S. G. F. Kaabi, F. Filali, "Channel Allocation and Routing in Wireless Mesh Networks: A survey and qualitative comparison between schemes," International Journal of Wireless and Mobile Nteworks (IJWMN), vol. 2, no. 1, pp., 2010.

[4] G. R. Hiertz, D. Denteneer, S. Max, R. Taori, J. Cardona, L. Berlemann, and B. Walke, "IEEE 802.11s: The WLAN Mesh Standard," IEEE Wireless Communications, vol. 17, no. 1, pp. 104-111, 2010.

[5] M. A. García, M. Angeles Santos, and J. Villalón, "IEEE 802.11n MAC Mechanisms for High Throughput: a Performance Evaluation," presented at the Seventh International Conference on Networking and Services, May 22, 2011, pp. 32 to 37.

[6] R. C. Carrano, Magalha, x, L. C. S. es, D. C. M. Saade, and C. V. N. Albuquerque, "IEEE 802.11s Multihop MAC: A Tutorial," IEEE Communications Surveys and Tutorials, vol. 13, no. 1, pp. 52-67, 2011.

[7] R. Garroppo, S. Giordano, D. Iacono, and L. Tavanti, "Notes on Implementing a IEEE 802.11s Mesh Point," in Wireless Systems and Mobility in Next Generation Internet, vol. 5122, Lecture Notes in Computer Science, L. Cerdà-Alabern, Ed.: Springer Berlin Heidelberg, 2008, pp. 60-72.

[8] G. R. Hiertz, S. Max, Z. Rui, D. Denteneer, and L. Berlemann, "Principles of IEEE 802.11s," presented at the 16th International Conference on Computer Communications and Networks, August 13-16, 2007, pp. 1002-1007. 
International Journal of Wireless \& Mobile Networks (IJWMN) Vol. 5, No. 3, June 2013

[9] D. Skordoulis, N. Qiang, C. Hsiao-Hwa, A. P. Stephens, L. Changwen, and A. Jamalipour, "IEEE 802.11n MAC frame aggregation mechanisms for next-generation high-throughput WLANs," IEEE Wireless Communications, vol. 15, no. 1, pp. 40-47, 2008.

[10] A. Ksentini, A. Guéroui, and M. Naimi, "Adaptive transmission opportunity with admission control for IEEE 802.11e networks," in the 8th ACM international symposium on Modeling, analysis and simulation of wireless and mobile systems. Montrial, Quebec, Canada: ACM, 2005, pp. 234-241.

[11] S. Ould Cheikh and A. Gueroui, "Multi-hop bandwidth reservation in WMN-based IEEE 802.11s (MBRWMN)," presented at the International Conference on Communications and Information Technology (ICCIT), June 26-28, 2012, pp. 211-215.

[12] D. B. Rawat, D. C. Popescu, and S. Min, "Performance enhancement of EDCA access mechanism of IEEE 802.11e wireless LAN," presented at the IEEE Radio and Wireless Symposium, January 22-24, 2008, pp. 507-510.

[13] H. Jie and M. Devetsikiotis, "Performance analysis of IEEE 802.11e EDCA by a unified model," presented at the IEEE Global Telecommunications Conference, November 29 - December 3, 2004, pp. 754-759.

[14] J. Hoblos, "Fairness enhancement in IEEE 802.11s multi-hop wireless mesh networks," presented at the 13th IEEE International Conference on Communication Technology (ICCT), Sept 25-28, 2011, pp. 647-651.

[15] K. Duffy, D. J. Leith, T. Li, and D. Malone, "Modeling 802.11 mesh networks," IEEE Communications Letters, vol. 10, no. 8, pp. 635-637, 2006.

[16] V. Mancuso, O. Gurewitz, A. Khattab, and E. W. Knightly, "Elastic Rate Limiting for Spatially Biased Wireless Mesh Networks," presented at the IEEE INFOCOM, March 14-19, 2010, pp. 1-9.

[17] J. Trivic, "Simulation of user plane in IEEE 802.11s mesh networks," presented at the 19th Telecommunications Forum (TELFOR), November 22-24, 2011, pp. 1624-1627.

[18] C. Sunghyun, J. del Prado, N. Sai Shankar, and S. Mangold, "IEEE 802.11 e contention-based channel access (EDCF) performance evaluation," presented at IEEE International Conference on Communications, Anchorage, AK, May 11-15, 2003, pp. 1151-1156.

[19] G. R. Hiertz, S. Max, T. Junge, D. Denteneert, and L. Berlemann, "IEEE 802.11s - Mesh Deterministic Access," presented at the 14th European Wireless Conference, June 22-25, 2008, pp. 18.

[20] C. Cicconetti, L. Lenzini, and E. Mingozzi, "Scheduling and Dynamic Relocation for IEEE 802.11s Mesh Deterministic Access," presented at the 5th annual IEEE Communications Society Conference on Sensor, Mesh and Ad Hoc Communications and Networks, June 16-20, 2008, pp. 19-27.

[21] T. Li, Ni,Qiang, Xiao,Yang, "Investigation of the block ACK scheme in wireless ad hoc networks," Wireless Communications and Mobile Computing, vol. 6, no. 6, pp. 877-888, 2006.

[22] L. Seungbeom and P. Sin-Chong, "Rotating Priority Queue based Scheduling Algorithm for IEEE 802.11n WLAN," The 9th International Conference on Advanced Communication Technology, vol. 3, no. 1702-1706, 2007.

[23] T. Hiatt and A. Prodan, "Investigating channel bonding and TXOP in 802.11n wireless networks," presented at the IEEE TENCON, Nov 21-24, 2011, pp. 435-439.

[24] O. Hoffmann and R. Kays, "Efficiency of frame aggregation in wireless multimedia networks based on IEEE 802.11n," presented at the 14th IEEE International Symposium on Consumer Electronics (ISCE), 7-10 June, 2010, pp. 1-5. 
International Journal of Wireless \& Mobile Networks (IJWMN) Vol. 5, No. 3, June 2013

[25] B. Ginzburg and A. Kesselman, "Performance analysis of A-MPDU and A-MSDU aggregation in IEEE 802.11n," presented at the IEEE Sarnoff Symposium, April 30 - May 2, 2007, pp. 1-5.

[26] A. Saif, M. Othman, S. Subramaniam, and N. Hamid, "An Enhanced A-MSDU Frame Aggregation Scheme for 802.11n Wireless Networks," Wireless Personal Communications, vol. 66, no. 4, pp. 683-706, 2012.

[27] O. Eng Hwee, J. Kneckt, O. Alanen, C. Zheng, T. Huovinen, and T. Nihtila, "IEEE 802.11ac: Enhancements for very high throughput WLANs," presented at the 22nd IEEE International Symposium on Personal Indoor and Mobile Radio Communications (PIMRC), September 11-14, 2011, pp. 849-853.

[28] K. Yaw-Wen, L. Tsern-Huei, H. Yu-Wen, and H. Jing-Rong, "Design and evaluation of a high throughput MAC with QoS guarantee for wireless LANs," presented at IEEE 9th Malaysia International Conference on Communications (MICC), December 15-17, 2009, pp. 869-873.

[29] M. Alicherry, R. Bhatia, and L. Li Erran, "Joint Channel Assignment and Routing for Throughput Optimization in Multiradio Wireless Mesh Networks," Journal on Selected Areas in Communications, vol. 24, no. 11, pp. 1960-1971, 2006.

[30] E. Khorov, A. Kiryanov, A. Lyakhov, and A. Safonov, "Analytical study of link management in IEEE 802.11s mesh networks," presented at the International Symposium on Wireless Communication Systems (ISWCS), August 28-31, 2012, pp. 786-790.

[31] Z. Wenxuan, W. Jing, and K. Guixia, "A novel High Throughput Long Training Field sequence design for next-generation WLAN," presented at Wireless Telecommunications Symposium (WTS), April 13-15, 2011, pp. 1-5.

[32] C.-Y. Wang and H.-Y. Wei, "IEEE 802.11n MAC Enhancement and Performance Evaluation," Mobile Networks and Applications, vol. 14, no. 6, pp. 760-771, 2009.

[33] K. Seongkwan, K. Youngsoo, C. Sunghyun, J. Kyunghun, and C. Jin-Bong, "A high-throughput MAC strategy for next-generation WLANs," presented at the 6th IEEE International Symposium on World of Wireless Mobile and Multimedia Networks, June 13-16, 2005, pp. 278-285.

[34] X. Wang and A. O. Lim, "IEEE 802.11s wireless mesh networks: Framework and challenges," Ad Hoc Networks, vol. 6, no. 6, pp. 970-984, 2008.

[35] T. Selvam and S. Srikanth, "A frame aggregation scheduler for IEEE 802.11n," presented at the National Conference on Communications (NCC), January 29-31, 2010, pp. 1-5.

[36] J. Camp and E. Knightly, "The IEEE 802.11s Extended Service Set Mesh Networking Standard," IEEE Communications Magazine, vol. 46, no. 8, pp. 120-126, 2008.

[37] I. Tinnirello and G. Bianchi, "Rethinking the IEEE 802.11e EDCA Performance Modeling Methodology," IEEE/ACM Transactions on Networking, vol. 18, no. 2, pp. 540-553, 2010.

[38] L. Bih-Hwang, C. Hung-Chi, and W. Huai-Kuei, "Study on multi-channel deterministic access for wireless mesh LAN," presented at the IEEE International Conference on Cyber Technology in Automation, Control, and Intelligent Systems (CYBER), May 27-31, 2012, pp. 39-42.

[39] R. G. Sanchez, H. Xiaojing, and C. Kwan-Wu, "Viability of concurrent transmission and reception for UWB radios over multipath channels," presented at the International Symposium on Communications and Information Technologies, October 17-19, 2007, pp. 1241-1246.

[40] Z. Yingnan, Z. Wenjun, L. Hang, G. Yang, and S. Mathur, "Supporting Video Streaming Services in Infrastructure Wireless Mesh Networks: Architecture and Protocols," presented at the IEEE International Conference on Communications, May 19-23, 2008, pp. 1850-1855. 
International Journal of Wireless \& Mobile Networks (IJWMN) Vol. 5, No. 3, June 2013

[41] P. Dely, A. Kassler, N. Bayer, and D. Sivchenko, "An Experimental Comparison of Burst Packet Transmission Schemes in IEEE 802.11-Based Wireless Mesh Networks," presented at the IEEE Global Telecommunications Conference, December 6-10, 2010, pp. 1-5.

[42] N. S. Nandiraju, D. S. Nandiraju, D. Cavalcanti, and D. P. Agrawal, "A novel queue management mechanism for improving performance of multihop flows in IEEE 802.11s based mesh networks," presented at the 25th IEEE International Performance, Computing, and Communications Conference, April 10-12, 2006, pp. 7 pp.-168.

[43] H. Ming-Xin and K. Geng-Sheng, "Delay and throughput Analysis of IEEE 802.11s Networks," presented at the IEEE International Conference on Communications Workshops, May 19-23, 2008, pp. 73-78.

[44] M. Singh, S.-G. Lee, W. Tan, and J. Lam, "Throughput Analysis of Wireless Mesh Network TestBed," in Convergence and Hybrid Information Technology, vol. 206, Communications in Computer and Info Science, G. Lee, D. Howard, and D. Ślęzak, Eds.: Springer Berlin Heidelberg, 2011, pp. 5461.

[45] B. Staehle, M. Bahr, F. Desheng, and D. Staehle, "Intra-Mesh Congestion Control for IEEE 802.11s Wireless Mesh Networks," presented at the 21st International Conference on Computer Communications and Networks (ICCCN), July 30 - August 2, 2012, pp. 1-7.

[46] L. Zheng, Y. Min, D. Heng, and D. Jufeng, "Concurrent Transmission Scheduling for Multi-Hop Multicast in Wireless Mesh Networks," presented at the 4th International Conference on Wireless Communications, Networking and Mobile Computing, October 12-14, 2008, pp. 1-4.

[47] M. Ozdemir, G. Daqing, A. B. McDonald, and Z. Jinyun, "Enhancing MAC Performance with a Reverse Direction Protocol for High-Capacity Wireless LANs," presented at the 64th IEEE Vehicular Technology Conference, September 25-28, 2006, pp. 1-5.

[48] D. Akhmetov, "802.11N: Performance Results of Reverse Direction Data Flow," presented at the IEEE 17th International Symposium on Personal, Indoor and Mobile Radio Communications, September 11-14, 2006, pp. 1-3. 